\title{
Association between habenula dysfunction and motivational symptoms in unmedicated major depressive disorder
}

\author{
Wen-Hua Liu, ${ }^{1,2}$ Vincent Valton, ${ }^{3}$ Ling-Zhi Wang, ${ }^{4}$ Yu-Hua Zhu, ${ }^{1}$ and \\ Jonathan P. Roiser ${ }^{3}$
}
${ }^{1}$ Department of Clinical Psychology, The Affiliated Brain Hospital of Guangzhou Medical University (Guangzhou Huiai Hospital), Guangzhou, China, ${ }^{2}$ School of Health Management, Guangzhou Medical University, Guangzhou, China, ${ }^{3}$ Institute of Cognitive Neuroscience, University College London, London, UK, and ${ }^{4}$ Department of Rehabilitation, The Affiliated Brain Hospital of Guangzhou Medical University (Guangzhou Huiai Hospital), Guangzhou, China

Correspondence should be addressed to Wen-Hua Liu, Department of Clinical Psychology, The Affiliated Brain Hospital of Guangzhou Medical University (Guangzhou Huiai Hospital), Guangzhou, China. E-mail: ahuatien@gmail.com

\begin{abstract}
The lateral habenula plays a central role in reward and punishment processing and has been suggested to drive the cardinal symptom of anhedonia in depression. This hypothesis is largely based on observations of habenula hypermetabolism in animal models of depression, but the activity of habenula and its relationship with clinical symptoms in patients with depression remains unclear. High-resolution functional magnetic resonance imaging (fMRI) and computational modelling were used to investigate the activity of the habenula during a probabilistic reinforcement learning task with rewarding and punishing outcomes in 21 unmedicated patients with major depression and 17 healthy participants. High-resolution anatomical scans were also acquired to assess group differences in habenula volume. Healthy individuals displayed the expected activation in the left habenula during receipt of punishment and this pattern was confirmed in the computational analysis of prediction error processing. In depressed patients, there was a trend towards attenuated left habenula activation to punishment, while greater left habenula activation was associated with more severe depressive symptoms and anhedonia. We also identified greater habenula volume in patients with depression, which was associated with anhedonic symptoms. Habenula dysfunction may contribute to abnormal response to punishment in patients with depression, and symptoms such as anhedonia.
\end{abstract}

Key words: depression; habenula; anhedonia; fMRI; punishment; prediction error

\section{Introduction}

There is strong evidence that the lateral habenula ( $\mathrm{LHb})$, which is anatomically and functionally connected to multiple structures that participate in reinforcement processing (Proulx et al., 2014), operates as a hub in a circuit that transforms motivational representations into appropriate behavioral outputs, especially for negatively valenced information. The habenula has recently attracted substantial interest due to the hypothesis that it may play an important role in certain symptoms of depression, such as low motivation. An extensive body of animal work supports this hypothesis, showing habenula 
hypermetabolism in rodent models of depression (Shumake and Gonzalez-Lima, 2013; Dillon et al., 2014; Lecca et al., 2014; Proulx et al., 2014; Benarroch, 2015; Zhao et al., 2015). However, the relationship between habenula function and symptoms of depression in humans remains largely unclear.

The habenula is thought to be involved in the brain's avoidance system mainly through encoding negative reward prediction errors, in the opposite direction than observed in dopamine neurons, which are excited by positive reward prediction errors (Matsumoto and Hikosaka, 2007, 2009a). Neurons in the LHb respond to unpleasant events such as reward omission and primary aversive stimuli (Matsumoto and Hikosaka, 2007; Hikosaka, 2010) and phasic changes in LHb neuronal activity contribute to subsequent aversive learning (Matsumoto and Hikosaka, 2011), decision making (Stopper and Floresco, 2014) and long-term aversive memory (Tomaiuolo et al., 2014). Excitatory outputs from the lateral habenula, mediated by the GABAergic rostromedial tegmental nucleus (RMTg), exert inhibitory control on midbrain dopaminergic neurons (Christoph et al., 1986; Ji and Shepard, 2007; Hong et al., 2011; Lammel et al., 2012; Stamatakis and Stuber, 2012). In addition, serotonergic input from the dorsal raphe nucleus (DRN) to the habenula also contributes to depressive-like behaviour in animal models (Zhao et al., 2015). How the dynamic interplay between these systems controls behavioral responses to aversive information and contributes to depressive behaviours is largely unknown.

It has been suggested that habenula dysfunction might be specifically linked to the development of anhedonia in depression (Dillon et al., 2014). Anhedonia, defined as a loss of interest or pleasure in previously enjoyable activities, is linked with disrupted transmission in the mesocorticolimbic dopamine system and is clinically important because it predicts poor response to standard antidepressant treatment (McMakin et al., 2012; Uher et al., 2012). Although there is currently no effective treatment specifically for anhedonia, suppression of habenula neuron firing has been shown to resolve depressive-like behaviour in rats (Li et al., 2011) and was reported to treat symptoms in a treatment-refractory patient through deep-brain stimulation (DBS) (Sartorius et al., 2010), suggesting a potential avenue for the development of novel therapeutic strategies. Anhedonia is also a feature of other psychiatric and neurological disorders, for example schizophrenia and Parkinson's disease, though its neurobiological basis may differ across pathologies (DerAvakian and Markou, 2012; Whitton et al., 2015). Understanding how the habenula drives negatively-motivated behavior may therefore lead to a better characterization of the neural mechanisms underlying symptoms of depression and other neuropsychiatric disorders.

Although the potential link between habenula dysfunction and depression is of great interest, very few studies have tested the contribution of habenula activity to symptoms in human patients, probably due to its small size (Lawson et al., 2013). Two studies reported that patients with major depression exhibited increased metabolic activity in the vicinity of the habenula during tryptophan depletion, measured during the resting state (Morris et al., 1999; Roiser et al., 2009). Habenula activation to aversive stimuli (cues associated with electric shocks) has recently been reported in healthy individuals with highresolution functional magnetic resonance imaging (fMRI) (Lawson et al., 2014; Hennigan et al., 2015) and habenula activation to negative feedback was reported in an earlier study, though using lower resolution image acquisition (Ullsperger and von Cramon, 2003). However, attenuated habenula activation was observed in patients with depression in two recent studies (Furman and Gotlib, 2016; Lawson et al., 2016), which is inconsistent with predictions from contemporary theories of its role in depression (Shumake and GonzalezLima, 2013; Proulx et al., 2014). Importantly, in these studies, which used a Pavlovian conditioning paradigm (Lawson et al., 2016) and a card guessing game (Furman and Gotlib, 2016), there was no clear contingency between the action made by participants and the outcome received, although actionoutcome associations are important for shaping adaptive responses to reinforcers. Hence, these previous studies were unable to address whether the habenula regulates instrumental learning, or how this may be disrupted in clinically depressed individuals.

Therefore, we used computational modelling and highresolution fMRI to investigate habenula function during an instrumental probabilistic reinforcement learning task in a sample of medication-free depressed individuals and healthy participants. The main goals were to investigate: (i) whether depressed individuals show reduced habenula activation during punishment processing on an instrumental task, and (ii) whether habenula activation is associated with clinical symptoms in patients with depression. Based on previous findings, we predicted reduced habenula activation during punishment processing in patients with depression, and that clinical measures of anhedonia would be negatively associated with habenula responses, over and above associations with general depressive symptoms.

\section{Materials and methods \\ Participants}

Twenty-four medication-free individuals with a diagnosis of major depressive disorder (15 females, age 19-50) were recruited from the outpatient clinic of a local psychiatric hospital (the Affiliated Brain Hospital of Guangzhou Medical University in China). The Structured Clinical Interview for DSM-IV (APA, 1994) was conducted by an experienced psychiatrist (L.Z.W or Y.H.Z) in the hospital to make the clinical diagnoses. All patients met the diagnostic criteria for major depressive disorder and were free from other Axis I disorders (other than anxiety) and psychotic features, life-time substance abuse/dependence, major medical or neurological illness, and current prescription of psychiatric medication (past 3 months). Disease severity at the time of scanning was quantified using the Hamilton Rating Scale for Depression (HDRS-24) (Hamilton, 1967), the Beck Depression Inventory II (BDI-II) (Beck et al., 1997) and the Beck Scale for Suicide Ideation (BSI) (Beck et al., 1979).

Twenty-one age-, education- and gender-matched healthy controls (12 females, age 22-45) were recruited by advertisement from the local community in Guangzhou. Healthy participants were screened for psychiatric conditions using a phone interview based on DSM-IV criteria. No healthy participants were taking any medication and none reported a history of psychiatric illness, neurological disease, major physical illness, substance or alcohol abuse, or mood/anxiety disorders in a first-degree relative. A cut-off score of 10 on the BDI-II (Beck et al., 1997) was used as a screening criterion.

The study was approved by the ethics committee of the Guangzhou Medical University and written informed consent was obtained from all participants. Six participants (three depressed participants and three healthy participants) who did not understand the task or failed to follow instructions, and one 
healthy individual with a clinically significant level of anhedonia [according to the criterion of $\geq 3$ points on the SHAPS defined by Snaith et al. (1995)] were excluded from all analyses. Healthy volunteers were paid 120 RMB for participating in the study, irrespective of the number of points won on the reinforcement learning task. Depressed patients were not paid for participation, but received a complimentary examination of their anatomical scan by a hospital radiologist.

\section{Reward learning task}

During fMRI, participants completed an instrumental probabilistic reward- and punishment-based associative learning task, adapted from those used in previous studies (Frank et al., 2004; van den Bos et al., 2012). Before scanning, participants were informed that they would be required to learn to associate specific stimuli with specific outcomes through trial and error selections, and were instructed to win as many points as possible (healthy volunteers were informed that their score would affect their payment, but in fact they were all paid the same amount). Four images (2 stimulus pairs, $A B$ and $C D$ : $A B$ was associated only with reward and $C D$ only with punishment) were presented, one pair on each trial, and participants were instructed to select one stimulus from each pair (e.g., A or B from AB) (Figure 1).

Participants were informed that, following their choices, they could win points, lose points, or receive 0 points (no feedback). Feedback was probabilistic: during reward trials (AB), when participants selected stimulus $A$, they received reward feedback ( +50 points and a green happy face) with $80 \%$ probability; when they selected stimulus $B$, they received reward feedback with $20 \%$ probability. On non-rewarded AB trials nooutcome feedback (a grey circle crossed diagonally by a line) was presented. During punishment trials (CD), when participants selected stimulus $\mathrm{C}$, they received punishment feedback ( -50 points and a red unhappy face) with $80 \%$ probability; when they selected stimulus $\mathrm{D}$, they received punishment feedback with $20 \%$ probability. On non-punished CD trials they received the same no-outcome feedback as on reward trials.

The trial sequence is displayed in Figure 1. On each trial, first either a green square (reward trials) or red square (punishment trials) was presented for $1000 \mathrm{~ms}$, followed by one of the stimulus pairs. Stimuli were displayed randomly on the left or the right side of the screen. Participants were required to choose either the left or the right stimulus within $2500 \mathrm{~ms}$. After the response was made, feedback was displayed for $1000 \mathrm{~ms}$. If no response was made within $2500 \mathrm{~ms}$, 'too slow' was presented on the screen for $1000 \mathrm{~ms}$. Finally, a blank screen was presented for 2500-RT (reaction time), making all trials the same length. A 500-6000 ms inter-trial jitter (fixation cross) was presented before the start of the next trial.

Participants completed 40 practice trials outside the scanner, using different stimulus pairs to the main task. The main task included 2 scanning runs of 100 trials each: 50 reward trials and 50 punishment trials per run, with a duration of approximately 10.5 min per run. Different sets of stimulus pairs were used in the first and the second runs.

\section{Behavioural data analysis}

Statistical analysis was performed using SPSS 19.0 software (SPSS Inc., Chicago, IL, USA). To test for group differences in task performance, reaction time (RT) and percentage choice of highprobability stimuli were analyzed using repeated-measures analysis of variance (ANOVA) with group (depressed, healthy) as the between-subjects factor and trial type (reward, punishment) as the within-subjects factor. Significant interaction effects were analyzed using post-hoc t-tests. Data that were not normally distributed were analysed using suitable nonparametric tests. Statistical significance was set at $P<0.05$, twotailed.

\section{Computational modelling of behavioural data}

Trial-by-trial analysis of behavioural choices was performed within a standard reinforcement learning (RL) framework (Sutton and Barto, 1998). Specifically, the RL framework assumes that subjects recursively learn and update the value of a given action (or stimulus) based on the average rewards received from previous exposure to that action and a prediction-error term. Formally, the expectation for future rewards of action ' $a$ ', ' $Q_{t+1}(a)$ ', is a function of the current expectation ' $\mathrm{Q}_{t}(a)$ ' and the difference between the actual reward that was experienced on the current trial ' $r$ ' (coded as 1,0 for rewards/punishments and no-rewards/no-punishments respectively) and the expected reward for trial ' $t$ ' (this discrepancy term is known as the prediction error-PE- $\left.\left[r_{t}-Q_{t}(a)\right]\right)$. The degree to which the PE updates the expectation is scaled by the learning rate parameter ' $\alpha$ ' (bounded between 0 and 1), such that:

$$
\mathrm{Q}_{\mathrm{t}+1}(a)=\mathrm{Q}_{\mathrm{t}}(a)+\alpha \times \underbrace{\left[r_{\mathrm{t}}-\mathrm{Q}_{\mathrm{t}}(a)\right]}_{\text {Prediction Error }}
$$

As a result, the lower the parameter ' $\alpha$ ', the longer a subject takes to acquire and update his expectations to the true underlying values of the actions. The probability that a subject chooses action ' $a$ ' on trial ' $t$ ', given the expected values of the available actions ' $Q_{t}(a)$ ' is given by the softmax link function:

$$
p_{t}\left[a \mid Q_{t}(a)\right]=\frac{e^{\left[Q_{t}(a) / \beta\right]}}{\sum_{a^{\prime}} e^{\left[Q_{t}\left(a^{\prime}\right) / \beta\right]}}
$$

The temperature parameter ' $\beta$ ' controls the choice stochasticity of the subject. That is, the extent to which a subject decides to choose the most rewarding option vs. exploring potentially more rewarding actions. A low ' $\beta$ ' would lead to consistent behavior, where the action with the highest expected value is invariably selected on each trial.

The model was then inverted and values for the ' $\alpha$ ' and ' $\beta$ ' parameters were estimated for each participant using the maximum a posteriori (MAP) procedure (Daw, 2011). This is a hierarchical Bayesian approach that allows for more accurate estimation of the true underlying parameters (Ahn et al., 2011), and is less sensitive to outliers than maximum likelihood estimation (MLE) (Daw, 2011). This two-stage procedure first estimates model parameters for each subject using MLE, and subsequently re-estimates the parameters of the model, this time applying priors on the possible range of parameters. The priors used during the MAP procedure are defined using Gaussian kernels with summary statistics extracted from the distribution of the ' $\alpha$ ' and ' $\beta$ ' parameters estimated using MLE. Computational modelling was implemented in Matlab R2016a (Mathworks), and used a nonlinear function optimization solver to recover the optimal parameters for each individual. Tests for group differences in the ' $\alpha$ ' and ' $\beta$ ' parameter estimates were performed using two sample t-tests $(P<0.05)$. 


\section{A reward}
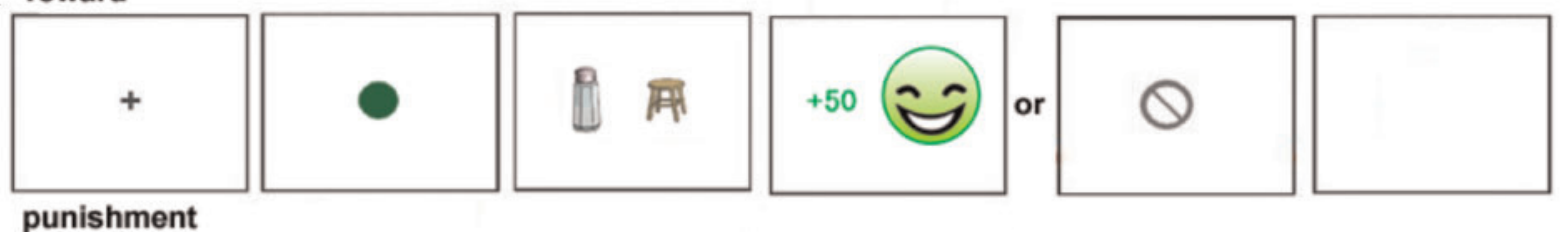

punishment
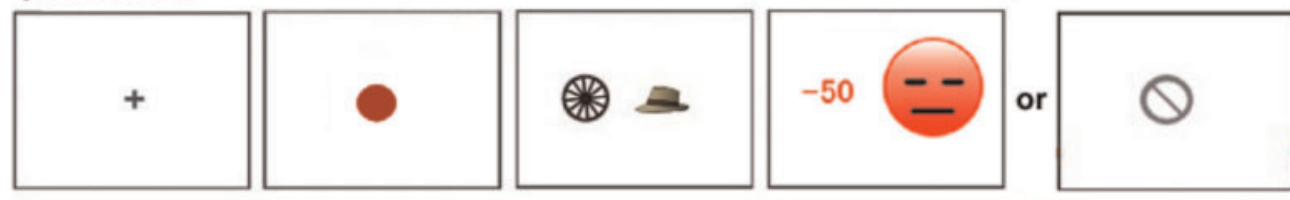

$500-6000 \mathrm{~ms}$

$1000 \mathrm{~ms}$

$\max 2500 \mathrm{~ms}$

$1000 \mathrm{~ms}$

2500ms - RT
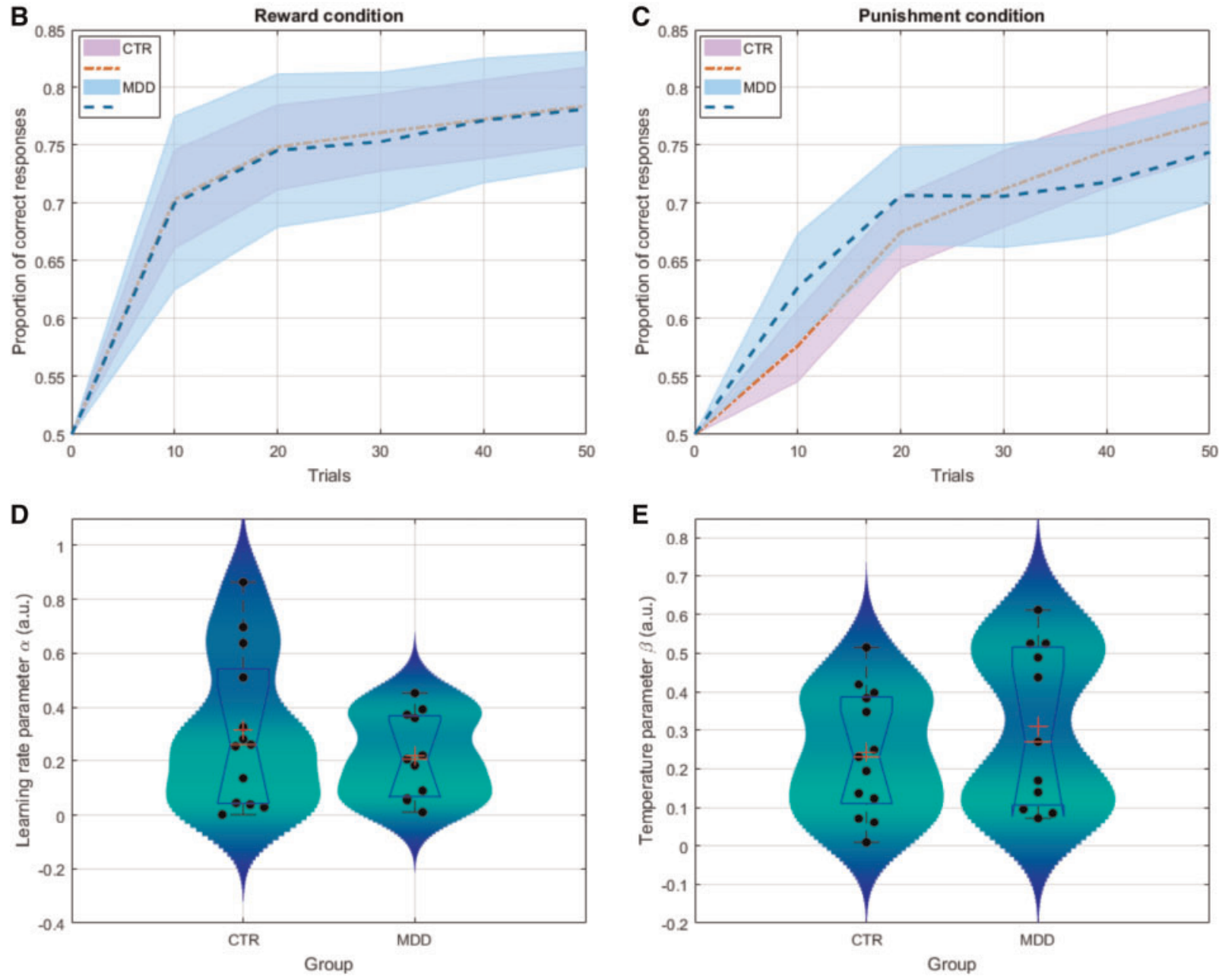

Fig. 1. Behavioral task and data. (A) Example of reward-based (top) and punishment-based (bottom) trials in the probabilistic associative learning task. Two pairs of stimuli were presented to the participants: (1) the reward (AB) pair provided $80 \%$ reward feedback for A and $20 \%$ for B; (2) the punishment (CD) pair provided $80 \%$ punishment feedback for C and 20\% for D. (B, C) Group-level performance over time in the reward and penalty conditions (average over the 2 blocks of 50 trials, binned every 10 trials). Dotted lines and shaded areas represent mean performance and standard error of the mean (SEM). (D, E) Comparison of learning rate parameters ( $\alpha$ ) and temperature parameters $(\beta)$ from the computational model. Box plots (blue) represent the distributions of parameters for each group (median is indicated by the red vertical line, whereas the mean is denoted by the large red cross). Violin plots (shaded blue-green area) represent the smoothed distribution of the data.

\section{Symptom assessments}

Anhedonic symptoms over the week preceding testing were measured using the SHAPS (Snaith et al., 1995), which contains 14 items. A higher score indicates more anhedonic symptoms. The Chinese version used for the present study has been shown to have adequate psychometric properties (Liu et al., 2012). Cronbach's alpha in the present sample was 0.89

The Temporal Experience of Pleasure Scale (TEPS) (Gard et al., 2006) was used to evaluate different components of trait pleasure processing, specifically the anticipatory and 
consummatory phases. A lower score indicates a higher level of anhedonia. The present study used a 20 -item Chinese version (Chan et al., 2010) that was modified from the original English version (18 items) (Gard et al., 2006). Cronbach's alphas for the TEPS-ANT (anticipatory pleasure) and the TEPS-CON (consummatory pleasure) in the present sample were 0.76 and 0.65 , respectively.

Symptoms of depression were measured using the BDI-II (Beck et al., 1997), assessed over the days immediately prior to testing. The scale contains 21 items and a higher score indicates greater severity of depression. Cronbach's alpha in the present sample was 0.96 .

Suicidal ideation was evaluated by the BSI which is a widely used instrument to assess thoughts, plans and intents relating to suicide over the last week (Beck et al., 1979). The scale includes 19 items and higher scores indicate greater suicidal ideation. Cronbach's alpha in the present sample was 0.94 .

\section{MRI acquisition and preprocessing}

Neuroimaging data were collected on a Philips 3.0 T Achieva scanner (Philips Healthcare, Inc., Best, Netherlands) equipped with an eight-channel SENSE (sensitivity encoding) head coil at the department of radiology in the local psychiatric hospital in Guangzhou. Functional images were acquired using a T2* weighted, echo planar single shot pulse sequence (repetition time (TR) $2000 \mathrm{msec}$; echo time (TE) $30 \mathrm{msec}$; flip angle $90^{\circ}$; matrix size $128 \mathrm{~mm} \times 128 \mathrm{~mm}$ (plane resolution using an $80 \times 80$ matrix, reconstructed to $128 \times 128$ ); field of view (FOV) $240 \mathrm{~mm} \times 240 \mathrm{~mm}$; SENSE factor 2; slice thickness $1.8 \mathrm{~mm}$; interslice gap, $0.2 \mathrm{~mm}$ ) with an in-plane acquisition resolution of $1.5 \times 1.5 \mathrm{~mm}$, during 2 functional runs of 312 volumes each. Whole-brain anatomical images were acquired using a sagittal T1-weighted magnetization-prepared rapid gradient echo scan (TR $11 \mathrm{msec}$; TE $5.7 \mathrm{msec}$; flip angle $8^{\circ}$; matrix size $240 \times 240 \mathrm{~mm}$; FOV $240 \mathrm{~mm} \times 240 \mathrm{~mm}$; resolution $0.75 \mathrm{~mm}$ isotropic; 220 slices). Thirty-seven coronal slices were acquired providing complete anterior-posterior coverage and inferiorsuperior coverage from the bottom of the temporal lobe to the top of the most dorsal part of the cingulate gyrus.

Data preprocessing was performed using Statistical Parametric Mapping software (SPM8: Wellcome Department of Cognitive Neurology, London). One healthy volunteer with a high level of head motion was excluded from all analyses. The first five scans were removed to allow for T1 equilibration. Echo planar images (EPIs) were corrected for differences in slice-time acquisition (to the middle slice) and realigned to the sixth volume of the first run.

For the habenula analysis, the EPIs were coregistered to each individual's anatomical scan [on which the habenula ROIs were drawn manually according to a previously described procedure (Lawson et al., 2013)], and smoothed using a 2-mm full-width at half-maximum (FWHM) Gaussian kernel. For the anatomical analysis, habenula size was calculated by computing the total volume within the left and right habenula ROIs.

For the whole-brain analysis, the EPIs were next spatially normalized to the Montreal Neurological Institute template and smoothed using a 6-mm FWHM Gaussian kernel. When specifying the statistical model (see below) the data were high pass filtered (cutoff $=128 \mathrm{~s}$ ), and temporal autocorrelations intrinsic to the fMRI time series were modelled using an AR(1) process.

\section{Neuroimaging data analysis}

We conducted two sets of analyses: the first was a standard event-related analysis to identify regions in which activation was modulated by reward and punishment processing; the second was a computational model-based analysis to identify regions in which activation was modulated by reward and punishment PEs. Data from 37 participants were utilized for the standard event-related analyses (21 depressed participants and 16 healthy participants).

For the computational model-based analysis, we compared our standard RL model to a variety of other models including (1) a null model, (2) a 'win-stay, lose-shift' model and (3) a more complex RL model that allowed the learning rate and temperature parameters to differ between reward and punishment conditions. These models were compared to our standard RL model using Bayesian Information Criterion scores (BIC), which test whether participants' behaviour is more parsimoniously explained by one model over others. The BIC compares models based on the number of free parameters and the likelihood of each model fit, where more parsimonious models with similar fits but fewer parameters are favoured (see Supplementary Figure S1).

Group level analyses revealed that participants' choices were most parsimoniously characterized by our standard RL model (the winning model showing decisive evidence$\Delta \mathrm{BIC}>100$, see Supplementary Figure S1). Model comparison was also performed at the individual subject and block level (i.e. 2 blocks of 50 trials per reward/punishment condition). At the individual subject level, the behavior of 10 patients and 3 controls was more parsimoniously described by the null model, suggesting that these participants were not responding on the task in accordance with the assumptions implicit to our model, thereby rendering their estimated parameters and predictionerrors uninterpretable. Consequently, these participants were excluded from the parametric computational modelling analysis, leaving data from 24 participants (11 depressed participants and 13 healthy controls). Out of the remaining participants, two patients and four controls had their parameters estimated from the 2nd block of choices only, as behavior on the first block favoured the null model in these subjects.

\section{Standard event-related analyses}

For the event-related analyses, at the first level a statistical model was computed by convolving a canonical hemodynamic response function with onsets time-locked to events of interest in order to create regressors. Four regressors were included: reward trials that received reward feedback; reward trials that received no-reward feedback; punishment trials that received punishment feedback; and punishment trials that received nopunishment feedback. Each trial was modeled as an epoch, time locked to the onset of the cue with duration equal to the entire trial (from the presentation of the cue to the disappearance of the outcome). The six realignment parameters were also included in the model. Where necessary, an additional regressor was included to model errors (i.e., trials with either invalid responses or on which no response was made $-0.66 \%$ of all trials). The inter-trial fixation cross was not modelled and constituted an implicit baseline.

Second-level analyses were conducted using the standard summary-statistics approach to random-effects analysis in SPM. Three contrast images were created for each participant through linear combination of the estimated beta images: (1) rewarded trials minus non-rewarded trials; (2) punished trials 
minus non-punished trials; (3) reward trials minus punishment trials. One-sample t-tests were conducted to identify regions activated by these contrasts across the whole sample, while two-sample t-tests were conducted to compare activation between the groups. The resulting images were thresholded at $P<0.001$, minimum cluster size 10 voxels. The statistical threshold was set at $P<0.05$, with whole brain family-wise error (FWE) correction for multiple comparisons (at the cluster level, unless otherwise stated).

For the habenula analysis, average contrast values were extracted from the habenula ROIs and entered into a repeatedmeasures ANOVA in SPSS, with group (depressed, healthy), hemisphere (right, left) and outcome (reward us neutral, punishment us neutral) as factors. Laterality was included in our analyses due to reports of asymmetry in the habenular complex in animal studies (Concha and Wilson, 2001; Husken et al., 2014). We also tested the association between habenula activation, habenula volume and depressive symptoms (SHAPS, TEPS and HDRS) using Pearson correlation coefficients.

\section{Model-based analyses}

We used our trial-by-trial computational model of task behavior in order to regress model components against trial-by-trial fluctuations in the BOLD response. Specifically, we examined activation corresponding to the magnitude of subject-level PEs (which were first standardized within each subject and condition) based on individual learning rates. We used the Q-learning algorithm described above (Watkins, 1989) to derive trial-bytrial PEs for the reward and punishment conditions separately, based on each participant's choices, feedback and learning rate.

At the first level of the computational model-based analyses, the presentation of the feedback screen was modeled as a stick function with zero duration. Reward and punishment trials were modelled in separate regressors (here with reward/nonreward trials modelled together and punishment/nonpunishment trials modelled together), and each was linearly parametrically modulated by its respective mean-corrected vector of PEs. The six realignment parameters were also included. Two contrast images were created for each participant: the reward $\mathrm{PE}$ regressor and the punishment $\mathrm{PE}$ regressor (note that, due to the continuous nature of the PE regressors, these intrinsically entail contrasts, and therefore do not require a comparison condition). At the group level, thresholding, correction for multiple comparisons and analysis of habenula ROIs were conducted as for the standard event-related analyses.

\section{Results}

\section{Demographic and clinical information}

Participant characteristics and symptom data are summarized in Table 1. The groups were matched for age, education and IQ. The depressed patients showed significantly higher levels of anhedonic symptoms (SHAPS and TEPS).

\section{Behavioral performance}

Behavioral data are presented in Table 2. For RT to choose highprobability stimuli, there was a marginally significant effect of trial type $\left(F(1,35)=3.53, P=0.07\right.$, partial $\left.\eta^{2}=0.09\right)$, with faster responses on reward trials relative to punishment trials. There was no main effect of group $(F(1,35)=0.39, P=0.53$, partial $\left.\eta^{2}=0.01\right)$ or trial type $\times$ group interaction $(F(1,35)=0.04, P=0.85$, partial $\eta^{2}=0.001$.
For percentage choice of high-probability stimuli, there was no main effect of trial type $(F(1,35)=1.85, P=0.18$, partial $\left.\eta^{2}=0.05\right)$, group $\left(F(1,35)=0.55, P=0.46\right.$, partial $\left.\eta^{2}=0.02\right)$ or trial type $\times$ group interaction $\left(F(1,35)=0.11, P=0.74\right.$, partial $\eta^{2}=$ 0.003). There was no significant difference between groups in the number of missing trials (Mann-Whitney $U, P=0.19$ ).

Analysis of parameter estimates from the computational modelling analysis (only conducted in subjects where our chosen model beat the null model) revealed that there was no significant difference between groups in the learning rate $(\alpha: \mathrm{t}(22)=0.42, \mathrm{P}=0.68)$ or choice stochasticity $(\beta: \mathrm{t}(22)=0.01$, $P=0.99)$.

\section{fMRI analysis}

Whole brain analysis-standard event-related. Within-group analyses showed that receipt of reward and punishment outcomes, relative to no-outcome, elicited activation in networks typically associated with reward and punishment processing in previous studies, including the ventral striatum (extending into the caudate), putamen and thalamus (Supplementary Table S1).

Between-group results showing regions associated with reward and punishment processing are summarized in Table 3. There were significant group differences in response to the receipt of punishment (us no outcome), with the depressed patients exhibiting significantly greater activation in the left substantia nigra $\left(x=-9, y=-18, z=-9, k=45, p_{\text {FWE }}<0.01\right.$, $Z=4.61)$, right superior colliculus $(x=6, y=-36, z=-9, k=61$, $\left.p_{\text {FWE }}<0.001, Z=4.55\right)$, left straight gyrus $(x=-9, y=18, z=-12$, $\left.\mathrm{k}=52, p_{\mathrm{FWE}}<0.01, Z=4.26\right)$, and right ventral striatum $(x=12$, $y=15, z=-6, k=29, p_{\text {FWE }}<0.05, Z=4.10$ ). No significant group differences in response to the receipt of reward (vs no-outcome) or learning condition (punishment us reward) were identified.

Whole brain analysis-parametric computational. Regions in which activation was modulated by PEs are summarized in Supplementary Tables S2 and S3. Across both groups, activation that scaled with the magnitude of punishment PEs was identified in the prefrontal cortex. The depressed patients exhibited significantly greater punishment PE-related activation in the right ventral striatum than the healthy volunteers $[x=6, y=9$, $z=-6, k=15, Z=4.82, p_{\mathrm{FWE}}<0.05$ (voxel-level)].

Habenula analysis-standard event-related. A repeated-measures ANOVA including group, trial type and hemisphere revealed a marginally significant interaction between group and hemisphere $\left(F(1,35)=3.76, P=0.06\right.$, partial $\left.\eta^{2}=0.10\right)$. The depressed group showed a trend towards greater activation across both trial types in the right habenula compared with the healthy controls $(t(35)=1.89, P=0.067$, Cohen's $d=0.65)$, but the difference in left habenula activation was non-significant $(t(35)=0.77$, $P=0.45$, Cohen's $d=0.26$ ). No other main effects or interactions approached significance (all ps >0.10) (Figure 2).

For comparability with a previous study (Furman and Gotlib, 2016) in which left-lateralised decreased habenula activation was identified in a depressed group, an analysis restricted to the left habenula was performed. Across both groups, there was a significant main effect of trial type $(F(1,35)=6.25, P=0.02$, partial $\left.\eta^{2}=0.15\right)$, with greater left habenula activation to punishment relative to reward $(t(36)=2.21, P=0.03$, Cohen's $d=0.51$ ). In addition, a trend towards a trial type $\times$ group interaction $\left(F(1,35)=3.31, P=0.077\right.$, partial $\left.\eta^{2}=0.09\right)$ was identified, which was driven by significantly greater activation in the left habenula during receipt of punishment than reward in the healthy 
Table 1. Participant characteristics

\begin{tabular}{|c|c|c|c|}
\hline & Patients with depression $(N=21)$ & Healthy controls $(N=17)$ & Analysis \\
\hline \multicolumn{4}{|l|}{ Demographic characteristics } \\
\hline Gender $(\mathrm{M} / \mathrm{F})$ & $9 / 12$ & $7 / 10$ & $\chi^{2}=0.05, P=0.82$ \\
\hline Age (years) & $30.7 \pm 8.9$ & $28.3 \pm 5.2$ & $t(36)=0.99, P=0.33$ \\
\hline Education (years) & $12.7 \pm 3.8$ & $13.6 \pm 2.0$ & $t(36)=1.02, P=0.31$ \\
\hline Estimated IQ & $96.6 \pm 17.9$ & $98.9 \pm 12.5$ & $t(36)=0.56, P=0.58$ \\
\hline \multicolumn{4}{|c|}{ Self-reported experience of symptoms } \\
\hline SHAPS (14-56) & $28.5 \pm 4.9$ & $23.6 \pm 4.8$ & $t(36)=3.09, P<0.01$ \\
\hline TEPS-ANT (11-66) & $38.5 \pm 8.2$ & $44.0 \pm 6.4$ & $t(36)=2.24, P<0.05$ \\
\hline TEPS-CON (9-54) & $34.0 \pm 6.2$ & $37.9 \pm 4.2$ & $t(36)=2.16, P<0.05$ \\
\hline TEPS total score & $72.6 \pm 14.0$ & $81.9 \pm 9.6$ & $t(36)=2.33, P<0.05$ \\
\hline \multicolumn{4}{|l|}{ Clinical characteristics } \\
\hline HDRS (baseline: 0-86) & $24.05 \pm 4.15$ & - & \\
\hline BSI (0-38) & $15.09 \pm 13.55$ & - & \\
\hline First episode of MDD & 13 & - & \\
\hline Past antidepressant use & 7 & - & \\
\hline Total number of episodes & $1.42 \pm 0.59$ & - & \\
\hline Age first episode (years) & $27.66 \pm 9.53$ & - & \\
\hline Duration of illness (years) & $2.90 \pm 2.65$ & - & \\
\hline
\end{tabular}

Data are presented as $n$ or mean $\pm \mathrm{SD}$.

SHAPS, Snaith-Hamilton Pleasure Scale; TEPS-ANT, Temporal Experience of Pleasure Scale - Anticipatory Pleasure Subscale; TEPS-CON, Temporal Experience of Pleasure Scale - Consummatory Pleasure Subscale; HDRS-24, Hamiton Depression Rating Scale-24 items; BSI, Scale for Suicide Ideation.

Table 2. Behavioural performance during fMRI

\begin{tabular}{lcc}
\hline & Patients with depression & Healthy controls \\
\hline Reward trials & & $72.5 \pm 15.6$ \\
$\quad$ Percent of choosing high-probability stimuli (\%) & $67.3 \pm 19.6$ & $767.3 \pm 169.4$ \\
$\quad$ Reaction time of choosing high-probability stimuli (ms) & $812.0 \pm 282.1$ & $71.0 \pm 15.9$ \\
Punishment trials & & $78.9 \pm 202.0$ \\
$\quad$ Percent of choosing high-probability stimuli (\%) & $65.0 \pm 16.2$ & $0.25 \pm 0.36$ \\
$\quad$ Reaction time of choosing high-probability stimuli (ms) & $831.2 \pm 288.9$ & $0.31 \pm 0.28$ \\
All trials & $1.26 \pm 1.92$ & $0.24 \pm 0.16$ \\
$\quad$ Missing trials $(\%)$ & $0.21 \pm 0.15$ & $0.31 \pm 0.21$ \\
$\quad$ Learning rate $(\alpha)$ & & \\
$\quad$ Choice stochasticity $(\beta)$ & & \\
\hline
\end{tabular}

Data are presented as mean $\pm \mathrm{SD}$.

controls only $(t(15)=3.35, P=0.004$, Cohen's $d=1.18)$. There was also a trend towards lower left habenula activation to receipt of punishment (relative to baseline) in the depressed group than the healthy controls $(t(35)=1.83, P=0.075$, Cohen's $d=0.62)$, but the group difference in activation during receipt of reward did not approach significance $(t(35)=0.90, P=0.37$, Cohen's $d=0.30$ ) (Figure 2).

Habenula analysis - parametric computational. Repeatedmeasures ANOVA with group, hemisphere and condition (reward-based PE, punishment-based PE) as factors revealed significant interactions between group and condition $(F(1,22)=$ 4.94, $P=0.037$, partial $\eta^{2}=0.18$ ). Consistent with the standard event-related analyses reported above, the healthy controls showed greater habenula activation during punishment relative to reward PE processing $(t(12)=3.72, P=0.003$, Cohen's $d=1.45)$, while this difference was non-significant in the depressed group ( $t(10)=0.36, P=0.72$, Cohen's $d=0.13$ ) (Figure 3 ).

\section{Relationship between habenula activation and clinical symptoms}

Due to low numbers of participants in the computational analysis, we did not analyse associations between clinical symptoms and PE-related activation.

In the depressed group, high levels of depressive symptoms (measured by the HDRS) were correlated with greater left habenula activation to the receipt of punishment $(r=0.51, P=0.019)$. High levels of anhedonia (measured by the TEPS-note that a lower score indicates higher anhedonia) were associated with greater left habenula activation during punishment relative to reward trials (TEPS: $r=-0.50, P=0.021$; TEPS-ANT: $r=-0.47$, $P=0.034$; TEPS-CON: $r=-0.51, P=0.02$; Figure 4). The correlations between anhedonia and left habenula activation remained significant (TEPS: $r=-0.50, p=0.025$; TEPS-ANT: $r=-0.47, P=0.035$; TEPS-CON: $r=-0.50, P=0.024$ ) after controlling for overall symptom severity (HDRS). No significant relationship was identified between anhedonia (measured by the SHAPS) and left habenula activation to the receipt of 
Table 3. Differences between the depressed $(N=21)$ and healthy control $(N=16)$ groups in neural responses to receipt of reward and punishment

\begin{tabular}{|c|c|c|c|c|c|c|}
\hline \multirow[b]{2}{*}{ Region $^{\mathrm{a}}$} & \multicolumn{3}{|c|}{ Coordinates $^{\mathrm{b}}$} & \multirow[b]{2}{*}{$k$} & \multirow[b]{2}{*}{ Z Score } & \multirow[b]{2}{*}{$P$ Value $^{\mathrm{C}}$} \\
\hline & $x$ & y & $z$ & & & \\
\hline \multicolumn{7}{|l|}{ Punishment us neutral } \\
\hline \multicolumn{7}{|l|}{ Depressed group $>$ controls } \\
\hline Left radiation of corpus callosum & -12 & 30 & 3 & 18 & 4.72 & $0.16^{\mathrm{d}}$ \\
\hline Left pars compacta of substantia nigra & -9 & -18 & -9 & 45 & 4.61 & $<0.01$ \\
\hline Right superior colliculus & 6 & -36 & -9 & 61 & 4.55 & $<0.001$ \\
\hline Left straight gyrus & -9 & 18 & -12 & 52 & 4.26 & $<0.01$ \\
\hline Right lateral accumbens & 12 & 15 & -6 & 29 & 4.10 & $<0.05$ \\
\hline Right area piriformis insulae & 21 & 9 & -18 & 22 & 4.09 & 0.09 \\
\hline Right fusiform gyrus & 30 & -42 & -21 & 19 & 3.91 & 0.14 \\
\hline Left anterior calcarine sulcus & -9 & -48 & 0 & 16 & 3.90 & 0.22 \\
\hline Left medial occipital gyrus & -36 & -81 & 21 & 19 & 3.89 & 0.14 \\
\hline Left lateral part of anterior orbital gyrus & -33 & 42 & -6 & 16 & 3.80 & 0.22 \\
\hline Right putamen & 24 & 18 & -3 & 13 & 3.77 & 0.33 \\
\hline Right posterior angular gyrus & 36 & -66 & 24 & 24 & 3.50 & 0.07 \\
\hline Right middle temporal gyrus & 57 & -57 & 6 & 12 & 3.35 & 0.38 \\
\hline \multicolumn{7}{|l|}{ Controls $>$ depressed group } \\
\hline \multicolumn{7}{|l|}{ Reward us neutral } \\
\hline \multicolumn{7}{|l|}{ Depressed group > controls } \\
\hline \multicolumn{7}{|l|}{ No clusters survived threshold } \\
\hline \multicolumn{7}{|l|}{ Controls > depressed group } \\
\hline No clusters survived threshold & & & & & & \\
\hline
\end{tabular}

Cluster-forming threshold $\mathrm{P}<0.001$ (uncorrected), minimum cluster size 10 voxels.

${ }^{a}$ Region is defined with reference to Atlas of the Human Brain (Mai et al., 2015).

${ }^{b}$ Montreal Neurological Institute coordinates.

${ }^{\mathrm{c}} \mathrm{P}<0.05$ cluster level (family-wise error corrected).

${ }^{\mathrm{d}} \mathrm{P}=0.03$ voxel level (family-wise error corrected).

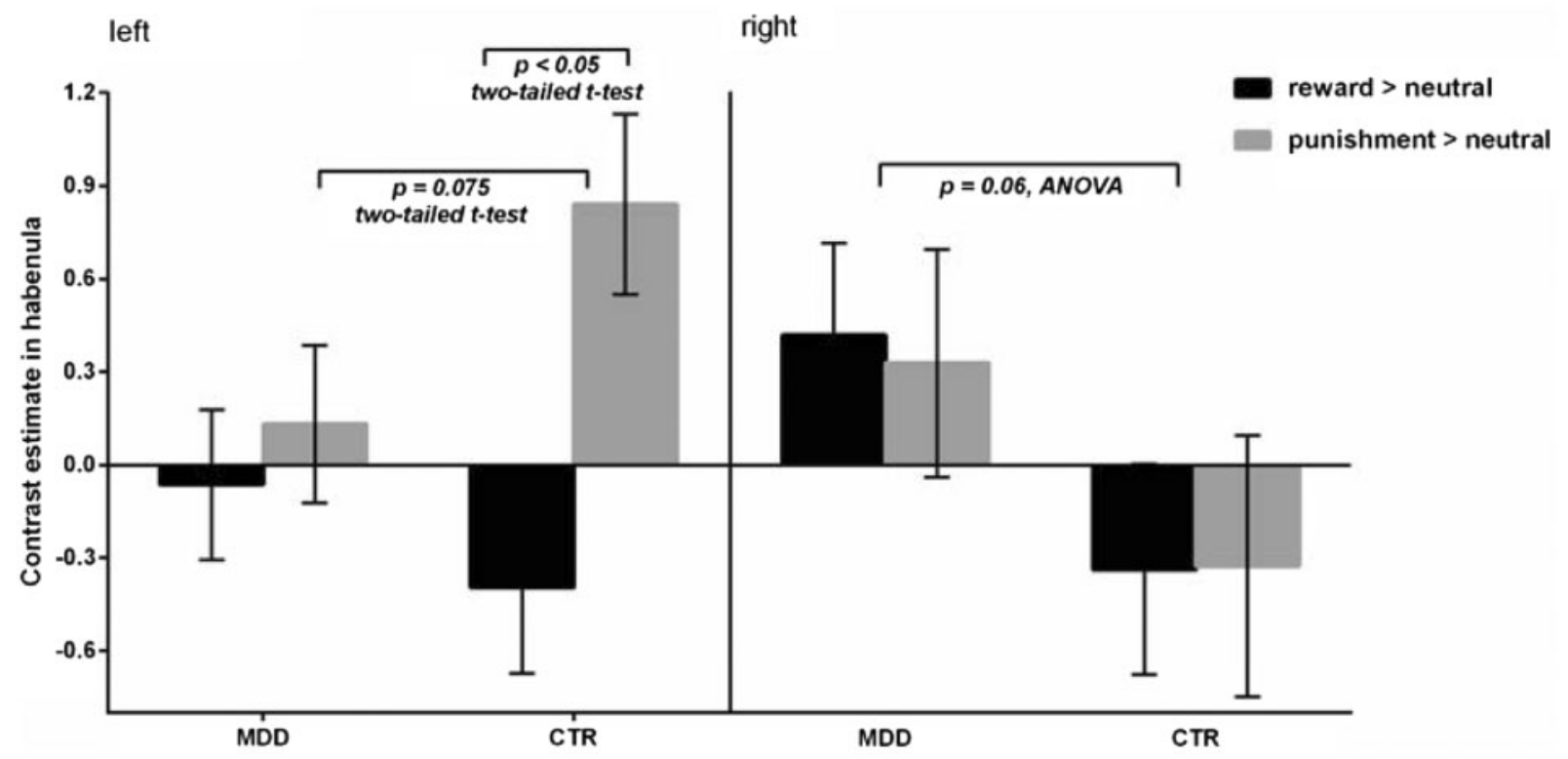

Fig. 2. Response in the left and right habenula to receipt of reward (reward $>$ neutral) and punishment (punishment $>$ neutral) in patients with major depressive disorder (MDD) and healthy controls (CTR). Error bars represent SEM. 


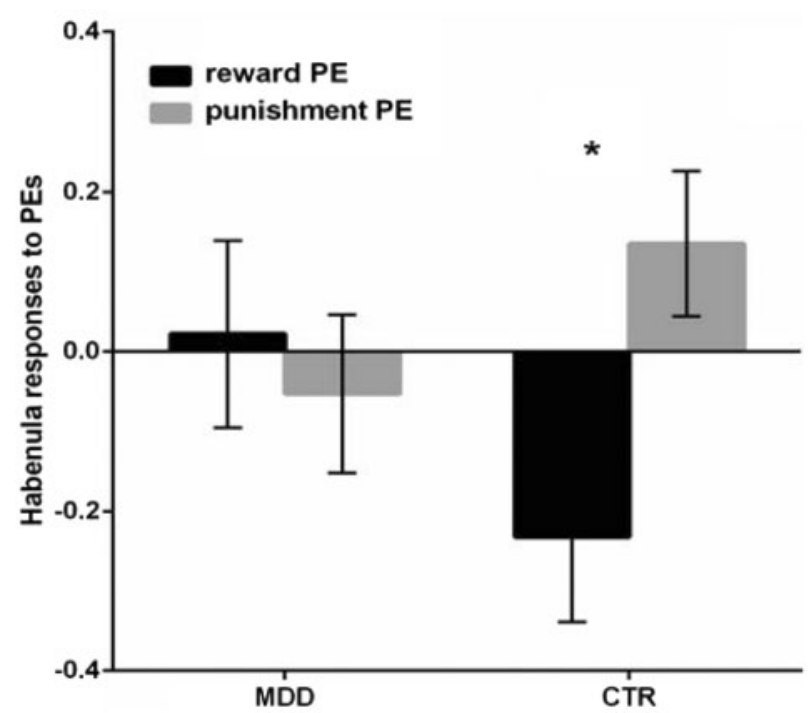

Fig. 3. Habenula responses to reward- and punishment-based prediction errors (PEs) in depressed patients and healthy controls. Error bars represent SEM.

punishment or during punishment relative to reward trials (all ps > 0.66; Supplementary Figure S2).

\section{Habenula volume and motivational symptoms}

Analysis of habenula volume (with group and hemisphere as factors) revealed significantly larger habenula volumes in the depressed group $\left(F(1,36)=7.20, P=0.01\right.$, partial $\left.\eta^{2}=0.17\right)$, but no main effect of hemisphere $\left(F(1,36)=0.80, P=0.38\right.$, partial $\eta^{2}=$ $0.02)$ or group-by-hemisphere interaction $(F(1,36)=0.50$, $P=0.48$, partial $\eta^{2}=0.01$ ) (Figure 5).

Larger normalized left habenula volumes were associated with greater anhedonia (TEPS: $r=-0.55, P=0.01$; TEPS-ANT: $r=-0.57$, $p<0.01$; TEPS-CON: $r=-0.48, P=0.03)$ in the depressed patients (Figure 4). These correlations remained significant (TEPS: $r=-0.55$, $P=0.01$; TEPS-ANT: $r=-0.58, P<0.01$; TEPS-CON: $r=-0.47$, $P=0.04)$ after controlling for overall symptom severity (HDRS). No significant associations between habenula volumes and anhedonic symptoms were identified in the healthy controls.

\section{Discussion}

This study explored the activation of the habenula during rewardand punishment-based learning in medication-free individuals with a diagnosis of major depressive disorder and healthy individuals. We also investigated the relationship between habenula activation, habenula volume and clinical symptoms.

Our results can be summarized as follows: (1) patients with depression showed increased activation during the receipt of punishment, but not reward outcomes, in several regions including the striatum; (2) receipt of punishment activated the left habenula in healthy individuals, in a manner consistent with PE processing; however, patients with depression showed a trend towards attenuated left habenula response to the receipt of punishment; (3) greater left habenula activation in depressed patients was associated with more severe depressive symptoms and anhedonia (assessed with the TEPS); and (4) patients with depression had greater habenula volumes, with a larger habenula associated with more anhedonic symptoms.

\section{Blunted habenula activation in depression during punishment processing}

Greater left habenula activation to the receipt of punishment relative to reward in healthy controls was identified, consistent with previous reports that the habenula conveys a negative reward signal during associative learning in humans (Lawson et al., 2014). This result is also consistent with findings in monkeys (Matsumoto and Hikosaka, 2007, 2009a). Similar to previous reports of dysfunctional habenula responses to punishment-related stimuli in patients with depression (Furman and Gotlib, 2016; Lawson et al., 2016), the present study also identified a trend towards attenuated left habenula response to the receipt of punishment in the patient group.

This finding could not be explained by differences in performing the reward learning task, in which behavior did not differ significantly between the groups. In addition, during the receipt of punishment, greater activation in several other reward-related brain regions was identified in depressed patients, including the left pars compacta of substantia nigra, the left straight gyrus (gyrus rectus), the right nucleus accumbens and the right superior colliculus. Given the extensive projections from these regions to the habenula (e.g., the substantia nigra directly regulates the excitation of habenula neurons [Gao et al., 1990; Gao et al., 1996; Matsumoto and Hikosaka, 2009b]), and the potentially important roles played by these structures in depression (e.g., the nucleus accumbens and straight gyrus have been considered as targets for deep brain stimulation (DBS) in treatment-resistant depression [Bewernick et al., 2010; Accolla et al., 2016]), these regions might contribute to abnormal habenula function in patients with depression.

A pattern of attenuated left habenula responses to punishment in patients with depression has been observed consistently in previous studies using a guessing game and a Pavlovian conditioning task (Furman and Gotlib, 2016; Lawson et al., 2016). One possible explanation is that this represents a failure to engage the habenula in the context of avoiding punishment. Such a failure to actively avoid punishment could potentially lead to hypersensitivity to negative outcomes in patients with depression. Consistent with this idea, previous behavioral and neuroimaging findings have suggested that patients with depression exhibited excessive sensitivity to negative feedback (Murphy et al., 2003; Taylor Tavares et al., 2008). A potential mechanism for this sensitivity could be enhanced activation of substantia nigra, given its known inhibitory inputs to the habenula (Matsumoto and Hikosaka, 2007); this explanation would be consistent with our whole-brain analysis, in which we observed greater activation in the depressed group in the substantia nigra during the receipt of punishment. Alternatively, a direct excitatory habenula-VTA pathway (Brown and Shepard, 2016) may also regulate the processing of aversive events in humans (Hennigan et al., 2015). However, whether this habenulaVTA pathway is involved in the processing of punishment in depressed patients remains unclear.

In this study, we identified significant group differences in punishment-related, but not in reward-related prediction errors. The reason for the lack of group differences in rewardrelated responses is unclear, but may relate to the specific design and analysis of our task, which was based on instrumental learning. This limits comparisons with previous studies of the neural basis of reward processing dysfunction in depression which have used tasks that do not require learning, e.g., the monetary incentive delay task (Knutson et al., 2008; Pizzagalli et al., 2009). Additionally, the inclusion of both reward and punishment conditions may have altered the saliency of the reward 

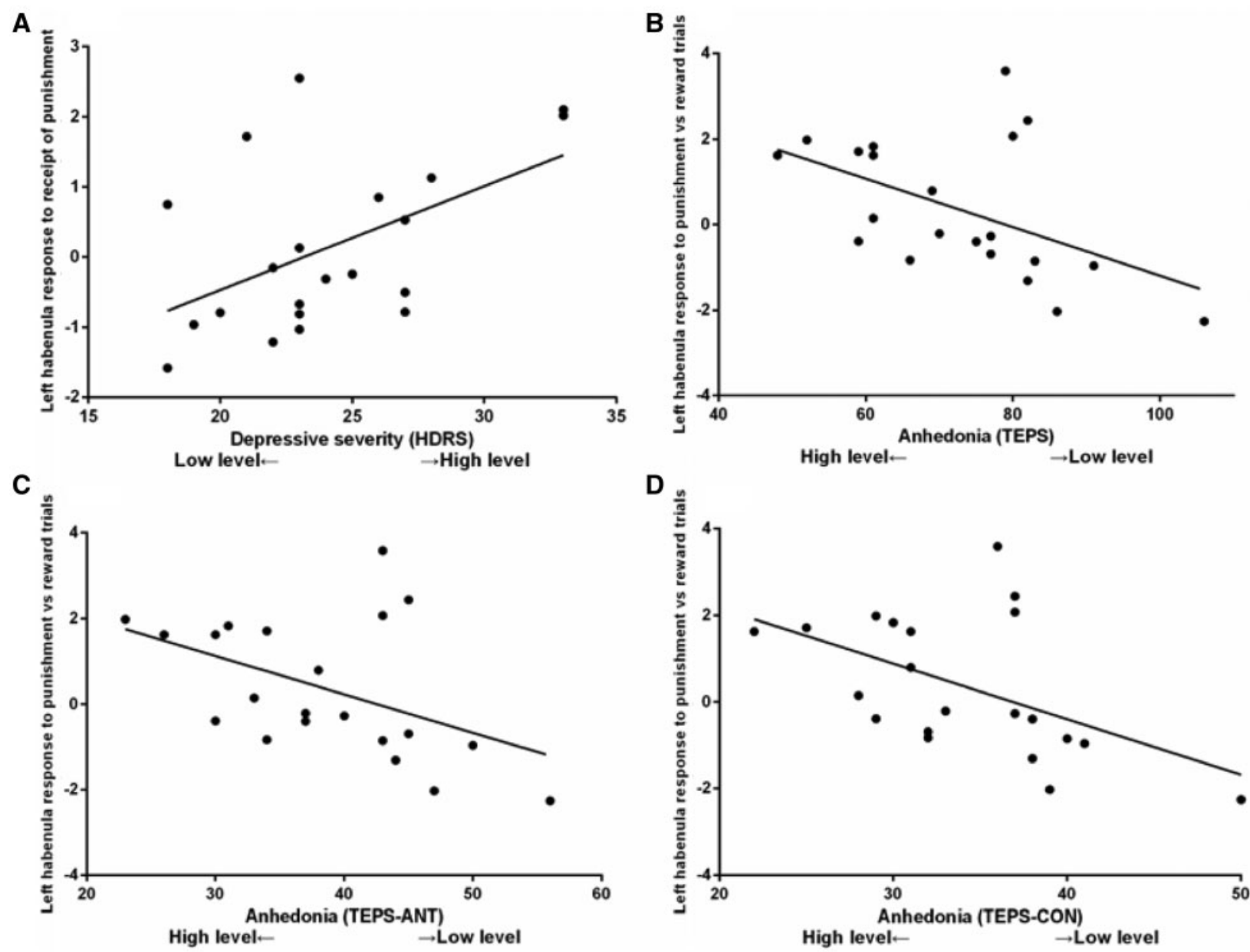

Fig. 4. Depressive symptoms (measured by the Hamilton Depression Rating Scale (HDRS)) and anhedonic symptoms (measured by the Temporal Experience of Pleasure Scale (TEPS), TEPS-ANT (anticipatory) and TEPS-CON (consummatory)) were associated with greater left habenula activation to the receipt of punishment us neutral outcomes, and greater left habenula response during punishment relative to reward trials, respectively, in depressed patients.

condition, and indeed similar tasks in healthy volunteers (Mattfeld et al., 2011) and in clinical patients (Palminteri et al., 2012) have demonstrated strong learning related differences between reward and punishment conditions. Our finding of group differences in the ventral stratum in response to the receipt of punishment (us no-outcome) and to punishment-based PE estimates is consistent with a recent study showing enhanced ventral stratum activation to punishment PEs in patients with depression (Ubl et al., 2015).

\section{Habenula activation and depressive symptoms}

The current study is the first to show that anhedonia is associated with habenula activation during punishment-based learning in patients with depression. Interestingly, and contrary to our hypothesis, anhedonia [which occurs more frequently in patients with severe symptoms (Pelizza and Ferrari, 2009)], was positively correlated with greater left habenula responses during punishment relative to reward processing, over and above associations with overall depressive symptoms. One possibility is that habenula dysfunction might influence patients' ability to differentially encode information relating to rewards and punishments. However, given the small sample tested here it would be premature to conclude that the association between anhedonic symptoms and habenula responses is due to either habenula hyperactivity to negative information or insensitivity to rewarding events. Although we detected significant associations between depressive symptoms and habenula responses, due to the relatively small sample size in our study it is possible that the correlation coefficients we report are inflated (with a mean $r$ value from significant tests of about 0.5) (Yarkoni, 2009).

The positive association between overall depressive symptoms and left habenula activation to the receipt of punishment in depressed patients is consistent with substantial evidence suggesting a relationship between habenula activity and depression-related behavior in rodents (Shumake and Gonzalez-Lima, 2013; Proulx et al., 2014; Zhao et al., 2015). Greater habenula activation in more severely depressed individuals might not result from attenuated (inhibitory) dopamine neuron input, but instead from a lower number of 5-HT neuron projections from the raphe nuclei, which have been proposed to be reduced in depression (Zhao et al., 2015). The only two studies to report greater tonic (i.e. resting-state) habenula activity in (remitted) depression used tryptophan depletion, which is thought to reduce brain 5-HT levels and can result in a temporary recurrence in depressive symptoms (Morris et al., 1999; Roiser et al., 2009). One of these studies also reported a stronger functional connection between the habenula and the dorsal raphe nucleus in patients with more severe symptoms following tryptophan depletion (Morris et al., 1999). To test this 

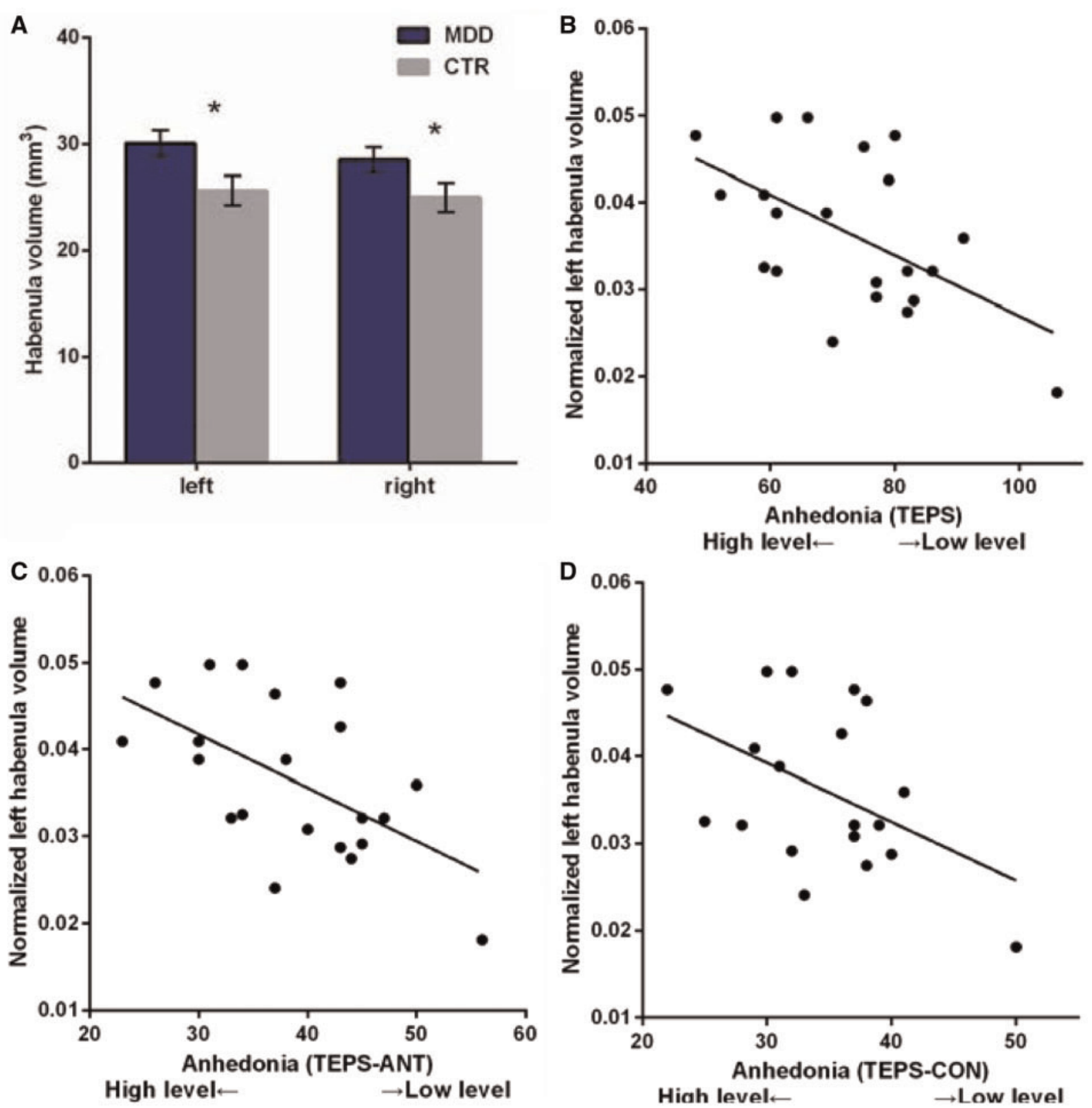

Fig. 5. (A) Habenula volumes in MDD and CTR. (B-D) Anhedonia (measured by TEPS, TEPS-ANT and TEPS-CON) was associated with larger normalized left habenula volume in patients. Error bars represent SEM.

hypothesis, it would be of great interest in future studies to measure habenula responses during reinforcement learning under conditions of tryptophan depletion.

Greater habenula volume in depression and association with symptoms

We also found greater habenula volumes in depressed patients, and a positive association between habenula volume and more severe anhedonic symptoms (measured by the TEPS). Consistent with our findings, greater habenula volume in depression has been reported in medicated women in their first-episode of illness (Carceller-Sindreu et al., 2015), and an association between greater habenula volume and overall depression severity (albeit not anhedonia specifically) was identified in unmedicated patients in a study at high field strength
(7T) (Schmidt et al., 2016). However, other studies have reported discrepant results. Although Lawson and colleagues (2016) also reported a positive association between habenula volume and overall depressive symptoms, again in unmedicated patients, they also observed a negative association between habenula volume and anhedonia (Lawson et al., 2016). Another study reported lower habenula volume only in depressed women (Savitz et al., 2011), though this was not replicated in subsequent study (Schmidt et al., 2016). The reasons for these discrepant results are unclear: differences between samples in medication status and/or symptom severity (Carceller-Sindreu et al., 2015; Schmidt et al., 2016) may be a factor. The present results suggest that there may be an increase in habenula volume in unmedicated severely depressed patients during the early stages of illness. However, this remains to be clarified in future studies. 


\section{Limitations}

Some limitations of our study merit comment. First, we enrolled acutely depressed patients with marked symptoms. This may limit the generalization of our findings to patients with milder symptoms. Second, our computational modelling analysis excluded several patients, due to behavior that suggested they were not engaging in the learning task in a manner consistent with our model; this limited our ability to perform meaningful correlations with symptoms for the computational analyses and to interpret these findings. Third, this study used secondary reinforcements, i.e., monetary gains and losses. Although there is some evidence showing that monetary losses are processed by the same brain circuitry as primary punishments such as electric shocks (Delgado et al., 2011), it will be important for future studies to compare the activity of habenula to primary and secondary punishments.

\section{Conclusions}

To our knowledge, this is the first study to explore habenula function in depression in the context of instrumental learning. We identified both structural and functional abnormalities in depressed patients: the habenula was larger in patients, especially in those with more severe anhedonic symptoms; and left habenula activation was attenuated during receipt of punishment, in a manner consistent with disrupted prediction error processing, although this pattern was less marked in those with more severe symptoms. Taken together, these results provide preliminary evidence that the habenula may contribute to symptoms of depression related to reinforcement learning such as anhedonia.

\section{Supplementary data}

Supplementary data are available at SCAN online.

\section{Funding}

This study was supported by grants from the National Natural Science Foundation of China (81501177) and China scholarship council (201508440192) to W.H.L. J.P.R. and V.V. were supported by the Wellcome Trust (101798/Z/13/Z). The funders had no role in study design, data collection and analysis, decision to publish, or preparation of the manuscript.

Conflict of interest. J.P.R. is a consultant for Cambridge Cognition and Takeda. The remaining authors do not have any conflicts of interest to be declared.

\section{References}

Accolla, E.A., Aust, S., Merkl, A., et al. (2016). Deep brain stimulation of the posterior gyrus rectus region for treatment resistant depression. Journal of Affective Disorders, 194, 33-7.

Ahn, W.Y., Krawitz, A., Kim, W., Busmeyer, J.R., Brown, J.W. (2011). A Model-based fMRI analysis with Hierarchical Bayesian parameter estimation. Journal of Neuroscience, Psychology, and Economics, 4, 95-110.

APA (1994). Diagnostic and Statistical Manual of Mental Disorders. Washington, DC: American Psychiatric Press.

Beck, A.T., Guth, D., Steer, R.A., Ball, R. (1997). Screening for major depression disorders in medical inpatients with the
Beck Depression Inventory for Primary Care. Behaviour Research and Therapy, 35, 785-91.

Beck, A.T., Kovacs, M., Weissman, A. (1979). Assessment of suicidal intention: the scale for suicide ideation. Journal of Consulting and Clinical Psychology, 47, 343-52.

Benarroch, E.E. (2015). Habenula: Recently recognized functions and potential clinical relevance. Neurology, 85, 992-1000.

Bewernick, B.H., Hurlemann, R., Matusch, A., et al. (2010). Nucleus accumbens deep brain stimulation decreases ratings of depression and anxiety in treatment-resistant depression. Biological Psychiatry, 67, 110-6.

Brown, P.L., Shepard, P.D. (2016). Functional evidence for a direct excitatory projection from the lateral habenula to the ventral tegmental area in the rat. Journal of Neurophysiology, jn 0030502016.

Carceller-Sindreu, M., de Diego-Adelino, J., Serra-Blasco, M., et al. (2015). Volumetric MRI study of the habenula in first episode, recurrent and chronic major depression. European Neuropsychopharmacology, 25, 2015-21.

Chan, R.C.K., Wang, Y., Huang, J., et al. (2010). Anticipatory and consummatory components of the experience of pleasure in schizophrenia: cross-cultural validation and extension. Psychiatry Research, 175, 181-3.

Christoph, G.R., Leonzio, R.J., Wilcox, K.S. (1986). Stimulation of the lateral habenula inhibits dopamine-containing neurons in the substantia nigra and ventral tegmental area of the rat. Journal of Neuroscience, 6, 613-9.

Concha, M.L., Wilson, S.W. (2001). Asymmetry in the epithalamus of vertebrates. Journal of Anatomy, 199, 63-84.

Daw, N.D. (2011).Trial-by-trial data analysis using computational models. In: Phelps, E. A., Robbins, T.W., Delgado, M. editors. Affect, Learning and Decision Making, Attention and Performance XXIII. New York: Oxford University Press.

Delgado, M.R., Jou, R.L., Phelps, E.A. (2011). Neural systems underlying aversive conditioning in humans with primary and secondary reinforcers. Frontiers in Neuroscience, 5, 71.

Der-Avakian, A., Markou, A. (2012). The neurobiology of anhedonia and other reward-related deficits. Trends in Neurosciences, $35,68-77$.

Dillon, D.G., Rosso, I.M., Pechtel, P., Killgore, W.D., Rauch, S.L., Pizzagalli, D.A. (2014). Peril and pleasure: an rdoc-inspired examination of threat responses and reward processing in anxiety and depression. Depression and Anxiety, 31, 233-49.

Frank, M.J., Seeberger, L.C., O'Reilly, R.C. (2004). By carrot or by stick: cognitive reinforcement learning in parkinsonism. Science, 306, 1940-3.

Furman, D.J., Gotlib, I.H. (2016). Habenula responses to potential and actual loss in major depression: preliminary evidence for lateralized dysfunction. Social Cognitive and Affective Neuroscience, 11, 843-51.

Gao, D.M., Hoffman, D., Benabid, A.L. (1996). Simultaneous recording of spontaneous activities and nociceptive responses from neurons in the pars compacta of substantia nigra and in the lateral habenula. European Journal of Neuroscience, 8, 1474-8.

Gao, D.M., Jeaugey, L., Pollak, P., Benabid, A.L. (1990). Intensitydependent nociceptive responses from presumed dopaminergic neurons of the substantia nigra, pars compacta in the rat and their modification by lateral habenula inputs. Brain Research, 529, 315-9.

Gard, D.E., Gard, M.G., Kring, A.M., John, O.P. (2006). Anticipatory and consummatory components of the experience of pleasure: A scale development study. Journal of Research in Personality, 40, 1086-102. 
Hamilton, M. (1967). Development of a rating scale for primary depressive illness. British Journal of Social and Clinical Psychology, 6, 278-96.

Hennigan, K., D’Ardenne, K., McClure, S.M. (2015). Distinct midbrain and habenula pathways are involved in processing aversive events in humans. Journal of Neuroscience, 35, 198-208.

Hikosaka, O. (2010). The habenula: from stress evasion to valuebased decision-making. Nature Reviews Neuroscience, 11, 503-13.

Hong, S., Jhou, T.C., Smith, M., Saleem, K.S., Hikosaka, O. (2011). Negative reward signals from the lateral habenula to dopamine neurons are mediated by rostromedial tegmental nucleus in primates. Journal of Neuroscience, 31, 11457-71.

Husken, U., Stickney, H.L., Gestri, G., et al. (2014). Tcf7l2 is required for left-right asymmetric differentiation of habenular neurons. Current Biology, 24, 2217-27.

Ji, H., Shepard, P.D. (2007). Lateral habenula stimulation inhibits rat midbrain dopamine neurons through a GABA(A) receptormediated mechanism. Journal of Neuroscience, 27, 6923-30.

Knutson, B., Bhanji, J.P., Cooney, R.E., Atlas, L.Y., Gotlib, I.H. (2008). Neural responses to monetary incentives in major depression. Biological Psychiatry, 63, 686-92.

Lammel, S., Lim, B.K., Ran, C., et al. (2012). Input-specific control of reward and aversion in the ventral tegmental area. Nature, 491, 212-7.

Lawson, R.P., Drevets, W.C., Roiser, J.P. (2013). Defining the habenula in human neuroimaging studies. Neuroimage, 64, 722-7.

Lawson, R.P., Nord, C.L., Seymour, B., et al. (2016). Disrupted habenula function in major depression. Molecular Psychiatry, 31, 81.

Lawson, R.P., Seymour, B., Loh, E., et al. (2014). The habenula encodes negative motivational value associated with primary punishment in humans. Proceedings of the National Academy of Sciences of the United States of America, 111, 11858-63.

Lecca, S., Meye, F.J., Mameli, M. (2014). The lateral habenula in addiction and depression: an anatomical, synaptic and behavioral overview. European Journal of Neuroscience, 39, 1170-8.

Li, B., Piriz, J., Mirrione, M., et al. (2011). Synaptic potentiation onto habenula neurons in the learned helplessness model of depression. Nature, 470, 535-9.

Liu, W.H., Wang, L.Z., Zhu, Y.H., Li, M.H., Chan, R.C. (2012). Clinical utility of the Snaith-Hamilton-pleasure scale in the Chinese settings. BMC Psychiatry, 12, 184.

Mai, J.K., Majtanik, M., Paxinos, G. (2015). Atlas of the Human Brain. San Diego: Academic Press/Elsevier.

Matsumoto, M., Hikosaka, O. (2007). Lateral habenula as a source of negative reward signals in dopamine neurons. Nature, 447, 1111-5.

Matsumoto, M., Hikosaka, O. (2009a). Representation of negative motivational value in the primate lateral habenula. Nature Neuroscience, 12, 77-84.

Matsumoto, M., Hikosaka, O. (2009b). Two types of dopamine neuron distinctly convey positive and negative motivational signals. Nature, 459, 837-41.

Matsumoto, M., Hikosaka, O. (2011). Electrical stimulation of the primate lateral habenula suppresses saccadic eye movement through a learning mechanism. PLoS One, 6, e26701.

Mattfeld, A.T., Gluck, M.A., Stark, C.E. (2011). Functional specialization within the striatum along both the dorsal/ventral and anterior/posterior axes during associative learning via reward and punishment. Learning and Memory, 18, 703-11.

McMakin, D.L., Olino, T.M., Porta, G., et al. (2012). Anhedonia predicts poorer recovery among youth with selective serotonin reuptake inhibitor treatment-resistant depression.
Journal of the American Academy of Child and Adolescent Psychiatry, 51, 404-11.

Morris, J.S., Smith, K.A., Cowen, P.J., Friston, K.J., Dolan, R.J. (1999). Covariation of activity in habenula and dorsal raphe nuclei following tryptophan depletion. Neuroimage, 10, 163-72.

Murphy, F.C., Michael, A., Robbins, T.W., Sahakian, B.J. (2003). Neuropsychological impairment in patients with major depressive disorder: the effects of feedback on task performance. Psychological Medicine, 33, 455-67.

Palminteri, S., Justo, D., Jauffret, C., et al. (2012). Critical roles for anterior insula and dorsal striatum in punishment-based avoidance learning. Neuron, 76, 998-1009.

Pelizza, L., Ferrari, A. (2009). Anhedonia in schizophrenia and major depression: state or trait?. Annals of General Psychiatry, 8, 22.

Pizzagalli, D.A., Holmes, A.J., Dillon, D.G., et al. (2009). Reduced caudate and nucleus accumbens response to rewards in unmedicated individuals with major depressive disorder. American Journal of Psychiatry, 166, 702-10.

Proulx, C.D., Hikosaka, O., Malinow, R. (2014). Reward processing by the lateral habenula in normal and depressive behaviors. Nature Neuroscience, 17, 1146-52.

Roiser, J.P., Levy, J., Fromm, S.J., et al. (2009). The effects of tryptophan depletion on neural responses to emotional words in remitted depression. Biological Psychiatry, 66, 441-50.

Sartorius, A., Kiening, K.L., Kirsch, P., et al. (2010). Remission of major depression under deep brain stimulation of the lateral habenula in a therapy-refractory patient. Biological Psychiatry, 67, e9-e11.

Savitz, J.B., Nugent, A.C., Bogers, W., et al. (2011). Habenula volume in bipolar disorder and major depressive disorder: a highresolution magnetic resonance imaging study. Biological Psychiatry, 69, 336-43.

Schmidt, F.M., Schindler, S., Adamidis, M., et al. (2016). Habenula volume increases with disease severity in unmedicated major depressive disorder as revealed by 7T MRI. European Archives of Psychiatry and Clinical Neuroscience, 12, 12.

Shumake, J., Gonzalez-Lima, F. (2013). Functional opposition between habenula metabolism and the brain reward system. Frontiers in Human Neuroscience, 7, 662.

Snaith, R.P., Hamilton, M., Morley, S., Humayan, A., Hargreaves, D., Trigwell, P. (1995). A scale for the assessment of hedonic tone the Snaith-Hamilton Pleasure Scale. The British Journal of Psychiatry, 167, 99-103.

Stamatakis, A.M., Stuber, G.D. (2012). Activation of lateral habenula inputs to the ventral midbrain promotes behavioral avoidance. Nature Neuroscience, 15, 1105-7.

Stopper, C.M., Floresco, S.B. (2014). What's better for me? Fundamental role for lateral habenula in promoting subjective decision biases. Nature Neuroscience, 17, 33-5.

Sutton, R.S., Barto, A.G. (1998). Reinforcement Learning, Cambridge (MA): MIT Press.

Taylor Tavares, J.V., Clark, L., Furey, M.L., Williams, G.B., Sahakian, B.J., Drevets, W.C. (2008). Neural basis of abnormal response to negative feedback in unmedicated mood disorders. Neuroimage, 42, 1118-26.

Tomaiuolo, M., Gonzalez, C., Medina, J.H., Piriz, J. (2014). Lateral Habenula determines long-term storage of aversive memories. Frontiers in Behavioral Neuroscience, 8, 170.

Ubl, B., Kuehner, C., Kirsch, P., Ruttorf, M., Diener, C., Flor, H. (2015). Altered neural reward and loss processing and prediction error signalling in depression. Social Cognitive and Affective Neuroscience, 10, 1102-12. 
Uher, R., Perlis, R.H., Henigsberg, N., et al. (2012). Depression symptom dimensions as predictors of antidepressant treatment outcome: replicable evidence for interest-activity symptoms. Psychological Medicine, 42, 967-80.

Ullsperger, M., von Cramon, D.Y. (2003). Error monitoring using external feedback: specific roles of the habenular complex, the reward system, and the cingulate motor area revealed by functional magnetic resonance imaging. Journal of Neuroscience, 23, 4308-14.

van den Bos, W., Cohen, M.X., Kahnt, T., Crone, E.A. (2012). Striatummedial prefrontal cortex connectivity predicts developmental changes in reinforcement learning. Cerebral Cortex, 22, 1247-55.
Whitton, A.E., Treadway, M.T., Pizzagalli, D.A. (2015). Reward processing dysfunction in major depression, bipolar disorder and schizophrenia. Current Opinion in Psychiatry, 28, 7-12.

Yarkoni, T. (2009). Big Correlations in Little Studies: Inflated fMRI Correlations Reflect Low Statistical Power-Commentary on Vul et al. (2009). Perspectives on Psychological Science, 4, 294-8.

Zhao, H., Zhang, B.-L., Yang, S.-J., Rusak, B. (2015). The role of lateral habenula-dorsal raphe nucleus circuits in higher brain functions and psychiatric illness. Behavioural Brain Research, 277, 89-98. 